\title{
Case Report \\ Postinfluenza Vaccination Idiopathic Thrombocytopenic Purpura in Three Elderly Patients
}

\author{
Joji Nagasaki, Masahiro Manabe, Kentaro Ido, Hiroyoshi Ichihara, Yasutaka Aoyama, \\ Tadanobu Ohta, Yoshio Furukawa, and Atsuko Mugitani
}

Department of Hematology, Seichokai Fuchu Hospital, 1-10-17 Hikocho, Izumi-shi, Osaka 594-0076, Japan

Correspondence should be addressed to Joji Nagasaki; j_nagasaki@seichokai.or.jp

Received 25 November 2015; Accepted 26 January 2016

Academic Editor: Gergely Feher

Copyright (C) 2016 Joji Nagasaki et al. This is an open access article distributed under the Creative Commons Attribution License, which permits unrestricted use, distribution, and reproduction in any medium, provided the original work is properly cited.

\begin{abstract}
The etiologies of secondary idiopathic thrombocytopenic purpura (ITP) include infection, autoimmune disease, and immunodeficiency. We report the cases of three elderly patients who developed ITP after receiving influenza vaccinations. The platelet count of an 81-year-old woman fell to $27,000 / \mu \mathrm{L}$ after she received an influenza vaccination. A 75 -year-old woman developed thrombocytopenia (5,000 platelets $/ \mu \mathrm{L})$ after receiving an influenza vaccination. An 87-year-old woman whose laboratory test values included a platelet count of $2,000 / \mu \mathrm{L}$ experienced genital bleeding after receiving an influenza vaccination. After Helicobacter pylori (HP) eradication or corticosteroid treatment, all of the patients' platelet counts increased. Influenza vaccination is an underlying etiology of ITP in elderly patients. HP eradication or corticosteroid treatment is effective for these patients. Clinicians should be aware of the association between ITP and influenza vaccinations.
\end{abstract}

\section{Introduction}

Idiopathic thrombocytopenic purpura (ITP) is an acquired form of thrombocytopenia caused by autoantibodymediated platelet destruction. The exact cause of ITP is unknown. It is initially treated with corticosteroids, intravenous immunoglobulin (IVIG), or intravenous anti-D immunoglobulin (IV anti-D). Corticosteroids are commonly recognized as a first-line therapy for acute ITP in adult patients. IVIG and IV anti-D are used for patients who need a rapid platelet increase or have a contraindication for corticosteroids. Although these treatments are effective and indispensable, the existence of treatment-related adverse events should be noted, especially in elderly patients. Here we report three elderly patients with ITP who had recently received influenza vaccinations. Their platelet counts increased after eradication of Helicobacter pylori (HP) or treatment with a corticosteroid.

\section{Case Reports}

2.1. Case 1. An 81-year-old woman whose platelet count was $184,000 / \mu \mathrm{L} 3$ months before admission was referred to our hospital because of thrombocytopenia. Her platelet count reduction was found unexpectedly during a medical examination; she had no symptoms caused by thrombocytopenia. She had received an influenza vaccination 4 weeks before developing thrombocytopenia. Her laboratory data at the time of thrombocytopenia diagnosis were as follows: platelet count, $39,000 / \mu \mathrm{L}$; white blood cell count, $4,900 / \mu \mathrm{L}$ without atypical cells; hemoglobin concentration, $14.1 \mathrm{~g} / \mathrm{dL}$; and platelet-associated IgG (PAIgG), $97 \mathrm{ng} / 10^{7}$ cells. Bone marrow was normocellular; the megakaryocyte count was $56 / \mu \mathrm{L}$. A urea breath test for HP was positive. The patient was diagnosed with ITP. HP was eradicated with $400 \mathrm{mg}$ of clarithromycin, 1,500 $\mathrm{mg}$ of amoxicillin hydrate, and $20 \mathrm{mg}$ of rabeprazole per day for 7 days. The patient's platelet count gradually increased and within 5 months had normalized. The patient's platelet count has remained within the reference range for over 2 years.

2.2. Case 2. A 75-year-old woman received an influenza vaccination; her platelet count measured the same day was $251,000 / \mu \mathrm{L}$. Five weeks after the vaccination, she was referred to our hospital because of thrombocytopenia with purpura 
combined with gingival and nasal hemorrhaging. Her laboratory data at admission were as follows: platelet count, $5,000 / \mu \mathrm{L}$; white blood cell count, $10,600 / \mu \mathrm{L}$ without atypical cells; hemoglobin concentration, $15.9 \mathrm{~g} / \mathrm{dL}$; and PAIgG, $3780 \mathrm{ng} / 10^{7}$ cells. Bone marrow was normocellular. A urea breath test for HP was positive and a diagnosis of ITP was established. She experienced massive bleeding because of marked thrombocytopenia; hence, we started HP eradication as well as steroid treatment $(1 \mathrm{mg} / \mathrm{kg}$ of prednisolone per day). Four weeks after the initiation of treatment, her platelet count had increased to within the normal range, so the steroid treatment was gradually tapered off over 2 months. No evidence of relapsed thrombocytopenia has been seen for over 15 months.

2.3. Case 3. An 87-year-old woman who showed no signs of platelet reduction such as purpura received an influenza vaccination. Two weeks after the vaccination, she was referred to us because of genital bleeding and purpura. Her laboratory data on admission were as follows: platelet count, $2,000 / \mu \mathrm{L}$; white blood cell count, $9,200 / \mu \mathrm{L}$ without atypical cells; hemoglobin concentration, $9.3 \mathrm{~g} / \mathrm{dL}$; and PAIgG, $950 \mathrm{ng} / 10^{7}$ cells. The bone marrow megakaryocyte count was $111 / \mu \mathrm{L}$. We diagnosed the patient with ITP and began treatment with $1 \mathrm{mg} / \mathrm{kg}$ of prednisolone per day. The steroid treatment resulted in a rapid increase in the patient's platelet count, so it was tapered off over several months. The patient exhibited no evidence of relapsed thrombocytopenia after treatment.

\section{Discussion}

These cases illustrate two facts: influenza vaccination caused ITP in three elderly patients, and HP eradication and/or corticosteroid administration may effectively treat postinfluenza vaccination ITP in elderly patients.

ITP is associated with several types of vaccinations. In previous studies, the risk of developing ITP increased after administration of measles-mumps-rubella (MMR), some together with MMR, hepatitis A, varicella, and diphtheriatetanus-pertussis vaccines in children and adolescents [12]. Adjuvants, which are compounds incorporated into vaccines to enhance immunogenicity, have been implicated in autoimmune/inflammatory syndrome induced by adjuvants (ASIA) [13]. Although thrombocytopenia can also be seen in ASIA patients, the association between ITP and influenza vaccination is still unclear. A number of case-control studies and case reports concerning postinfluenza vaccination ITP have been published (Table 1) [1-11, 14]. The Berlin CaseControl Surveillance Study of drug-associated ITP concluded that influenza vaccinations increase the risk of ITP. Twelve cases of postinfluenza vaccination ITP, including our three, have been reported. Features common to most reported cases include PAIgG elevation, time from vaccination to development of ITP, and treatment response.

All of our patients and most patients in previous reports had PAIgG elevations. PAIgG can detect both surface IgG and $\alpha$-granule IgG following platelet lysis [15]. Vaccine-associated ITP is probably caused by molecular mimicry, which involves the activation of autoreactive $\mathrm{B}$ or $\mathrm{T}$ cells by peptides in the vaccine that exhibit structural similarity to antigens found on platelets [12]. Elevated PAIgG may be an antibody produced by molecular mimicry.

Two of our patients developed ITP within 4 weeks after influenza vaccination and the third within 5 weeks. In previous patient reports, postinfluenza vaccination ITP developed 4-26 days after vaccination. This variability may depend on the patient's influenza antibody status. Some patients may have preexisting antibodies from prior influenza vaccines, some may be mounting an anamnestic response to a previously encountered antigen, and others may be undergoing primary alloimmunization because they had no previous exposure to the antigen [16]. An anamnestic response occurs in 3-10 days. Primary alloimmunization requires at least 23 weeks. Therefore, postinfluenza vaccination ITP in elderly patients may occur within several days or 2-3 weeks after vaccination.

In a previous report, elderly patients with ITP had a response rate of about $60 \%$ and were likely to relapse after treatment (31.3\% of patients relapsed) [17]. In previously reported cases of postinfluenza vaccination ITP, 8 of 9 patients made a full recovery (Table 1). All three of our patients also achieved platelet recovery and were stable after treatment. Therefore, postinfluenza vaccination ITP in elderly patients may respond to treatment.

MMR vaccine is associated with the development of ITP in children [18]. The clinical features are similar to those of postinfluenza vaccination ITP. Signs usually occur within 6 weeks after vaccination [19], and more than $90 \%$ of patients recover from thrombocytopenia within 6 months after diagnosis [20]. In contrast to post-MMR vaccination ITP, 7 of the 12 reported postinfluenza vaccination ITP patients were elderly (Table 1), possibly because influenza vaccinations are recommended for elderly patients. ITP is likely to be found in elderly patients because they exhibit bleeding symptoms more frequently [21]. Two of our three patients with ITP were admitted to the hospital with bleeding symptoms.

HP eradication, corticosteroid administration, or both may effectively treat postinfluenza vaccination ITP in elderly patients. Prednisone, IVIG, and IV anti-D are recommended general treatments for ITP patients with severe thrombocytopenia or bleeding [22]. The rate of treatment-related adverse events, such as IVIG-related acute renal failure and steroid-induced diabetes or infection, is higher in elderly than in young patients with ITP [21]. Our patients' platelet counts increased smoothly without the use of IVIG and with no adverse events. One patient achieved full recovery and longterm remission with HP eradication only. We treated two patients with corticosteroids because of their bleeding symptoms. Post-MMR vaccination ITP is associated with a shorter duration of thrombocytopenia and less severe bleeding than is primary ITP [20]. For patients with postinfluenza vaccination ITP as well as those with primary ITP, corticosteroids and HP eradication may be effective treatments. HP eradication may be an effective and safe treatment for elderly patients with postinfluenza vaccination ITP, especially those without severe bleeding. 


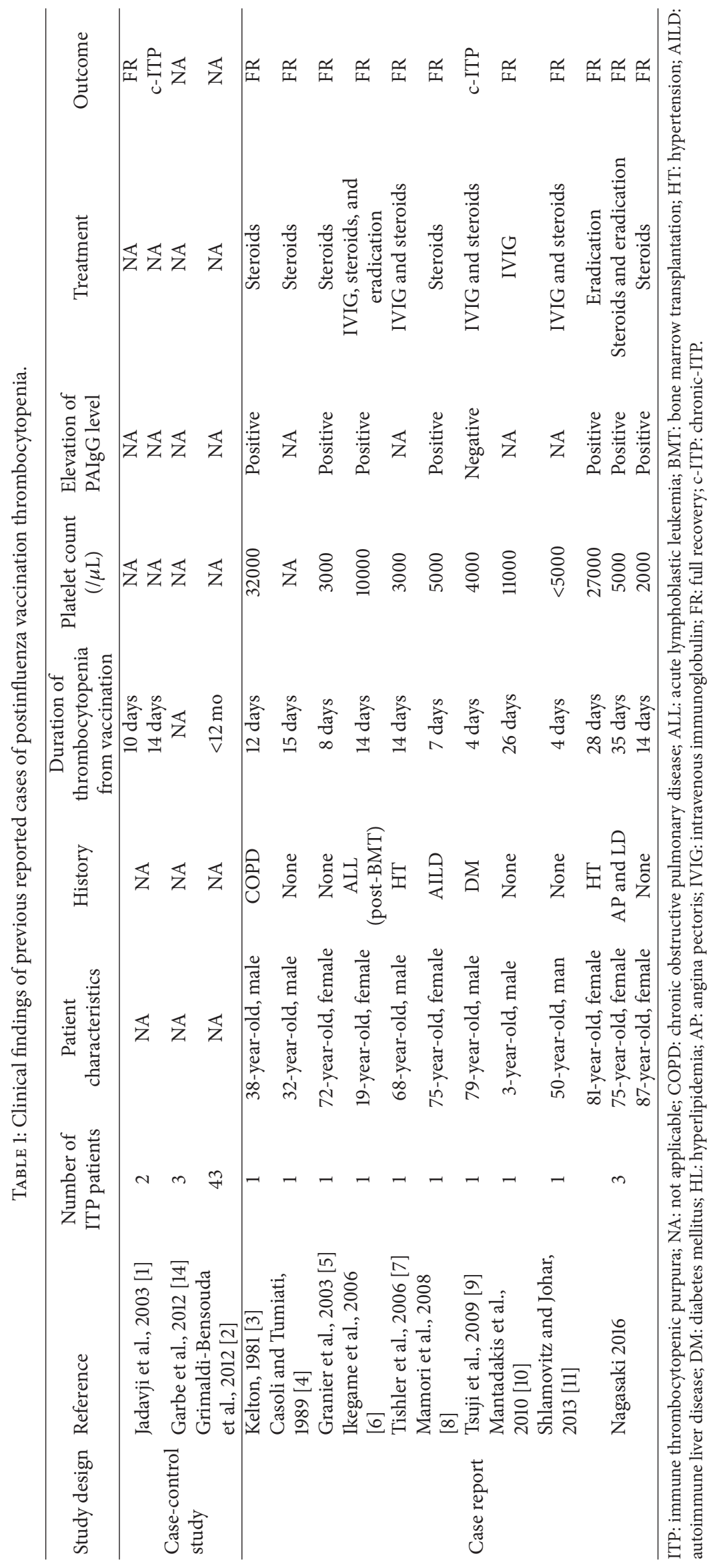


In conclusion, while influenza vaccinations are useful for preventing influenza infections and their complications, clinicians should be aware that an association with ITP is strongly suspected.

\section{Conflict of Interests}

The authors declare that there is no conflict of interests regarding the publication of this paper.

\section{References}

[1] T. Jadavji, D. Scheifele, and S. Halpein, "Thrombocytopenia after immunization of Canadian children, 1992 to 2001," The Pediatric Infectious Disease Journal, vol. 22, no. 2, pp. 119-122, 2003.

[2] L. Grimaldi-Bensouda, M. Michel, E. Aubrun et al., "A casecontrol study to assess the risk of immune thrombocytopenia associated with vaccines," Blood, vol. 120, no. 25, pp. 4938-4944, 2012.

[3] J. G. Kelton, "Vaccination-associated relapse of immune thrombocytopenia," The Journal of the American Medical Association, vol. 245, no. 4, pp. 369-370, 1981.

[4] P. Casoli and B. Tumiati, "Acute idiopathic thrombocytopenic purpura after anti-influenza vaccination," Medicina, vol. 9, pp. 417-418, 1989 (Italian).

[5] H. Granier, X. Nicolas, J.-P. Laborde, and F. Talarmin, "Severe autoimmune thrombopenia following anti-influenza vaccination," Presse Medicale, vol. 32, no. 26, pp. 1223-1224, 2003 (French).

[6] K. Ikegame, K. Kaida, T. Fujioka et al., "Idiopathic thrombocytopenic purpura after influenza vaccination in a bone marrow transplantation recipient," Bone Marrow Transplantation, vol. 38, no. 4, pp. 323-324, 2006.

[7] M. Tishler, O. Levy, and M. Amit-Vazina, "Immune thrombocytopenic purpura following influenza vaccination," Israel Medical Association Journal, vol. 8, no. 5, pp. 322-323, 2006.

[8] S. Mamori, K. Amano, H. Kijima, I. Takagi, and H. Tajiri, "Thrombocytopenic purpura after the administration of an influenza vaccine in a patient with autoimmune liver disease," Digestion, vol. 77, no. 3-4, pp. 159-160, 2008.

[9] T. Tsuji, H. Yamasaki, and H. Tsuda, "Refractory idiopathic thrombocytopenic purpura following influenza vaccination," Rinshō Ketsueki, vol. 50, no. 7, pp. 577-579, 2009 (Japanese).

[10] E. Mantadakis, E. Farmaki, S. Thomaidis, A. Tsalkidis, and A. Chatzimichael, "A case of immune thrombocytopenic purpura after influenza vaccination: consequence or coincidence?" Journal of Pediatric Hematology/Oncology, vol. 32, no. 6, pp. e227e229, 2010.

[11] G. Z. Shlamovitz and S. Johar, "A case of Evans' syndrome following influenza vaccine," Journal of Emergency Medicine, vol. 44, no. 2, pp. el49-e151, 2013.

[12] M. Rinaldi, C. Perricone, O.-D. Ortega-Hernandez, R. Perricone, and Y. Shoenfeld, "Immune thrombocytopaenic purpura: an autoimmune cross-link between infections and vaccines," Lupus, vol. 23, no. 6, pp. 554-567, 2014.

[13] P. Pellegrino, E. Clementi, and S. Radice, "On vaccin's adjjuvants and autoimmunity-current evidence and future perspectives," Autoimmunity Reviews, vol. 14, pp. 880-888, 2015.
[14] E. Garbe, F. Andersohn, E. Bronder et al., "Drug-induced immune thrombocytopaenia: results from the Berlin CaseControl Surveillance Study," European Journal of Clinical Pharmacology, vol. 68, no. 5, pp. 821-832, 2012.

[15] J. N. George, "Platelet immunoglobulin G: its significance for the evaluation of thrombocytopenia and for understanding the origin of $\alpha$-granule proteins," Blood, vol. 76, no. 5, pp. 859-870, 1990.

[16] B. Kenney and G. Stack, "Drug-induced thrombocytopenia," Archives of Pathology and Laboratory Medicine, vol. 133, no. 2, pp. 309-314, 2009.

[17] H. Zhou, R. Fu, H. Wang et al., "Immune thrombocytopenia in the elderly: clinical course in 525 patients from a single center in China," Annals of Hematology, vol. 92, no. 1, pp. 79-87, 2013.

[18] V. Cecinati, N. Principi, L. Brescia, P. Giordano, and S. Esposito, "Vaccine administration and the development of immune thrombocytopenic purpura in children," Human Vaccines \& Immunotherapeutics, vol. 9, no. 5, pp. 1158-1162, 2013.

[19] T. Jefferson, D. Price, V. Demicheli, and E. Bianco, "Unintended events following immunization with MMR: a systematic review," Vaccine, vol. 21, no. 25-26, pp. 4135-4136, 2004.

[20] V. Cecinati, N. Principi, L. Brescia, P. Giordano, and S. Esposito, "Vaccine administration and the development of immune thrombocytopenic purpura in children," Human Vaccines and Immunotherapeutics, vol. 9, no. 5, pp. 1158-1162, 2013.

[21] M. Michel, O. B. Rauzy, F. R. Thoraval et al., "Characteristics and outcome of immune thrombocytopenia in elderly: results from a single center case-controlled study," American Journal of Hematology, vol. 86, no. 12, pp. 980-984, 2011.

[22] D. B. Cines and J. B. Bussel, "How I treat idiopathic thrombocytopenic purpura (ITP)," Blood, vol. 106, no. 7, pp. 2244-2251, 2005 . 


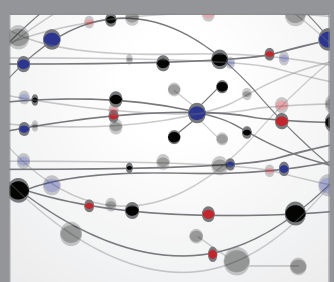

The Scientific World Journal
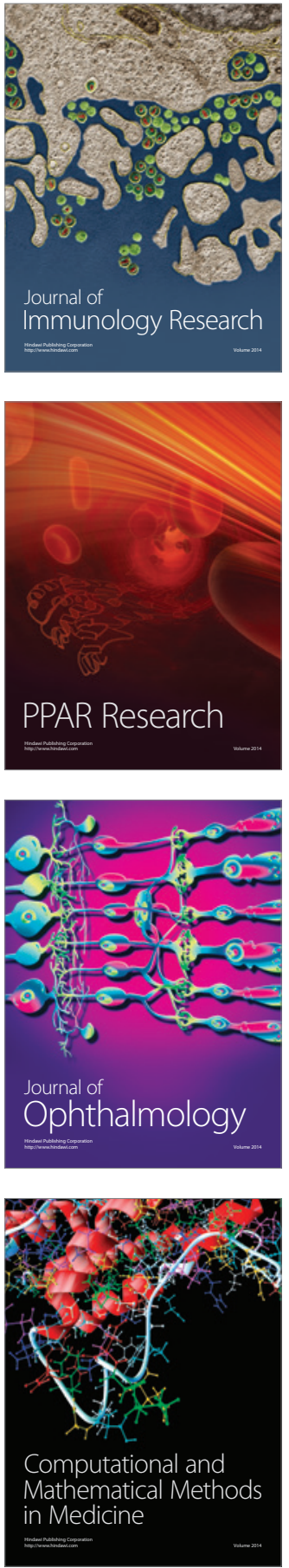

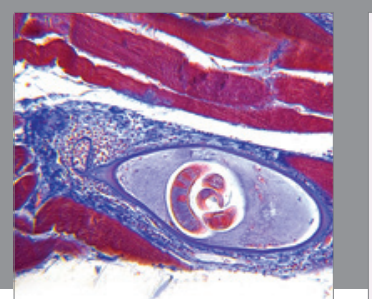

Gastroenterology Research and Practice

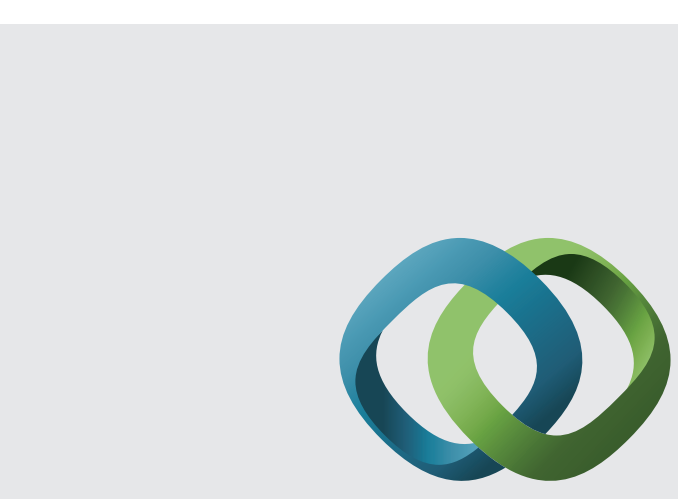

\section{Hindawi}

Submit your manuscripts at

http://www.hindawi.com
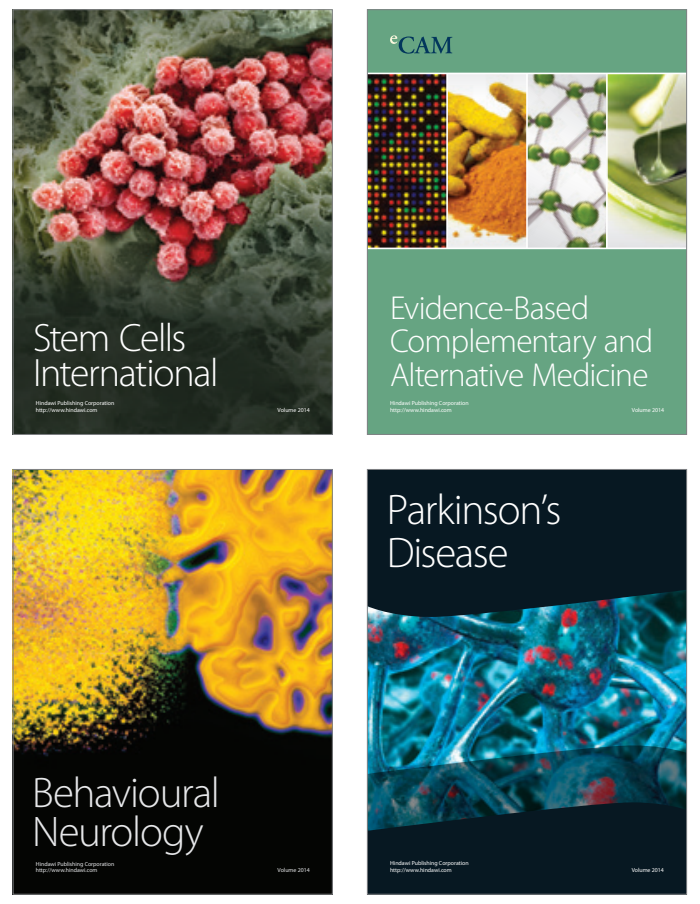
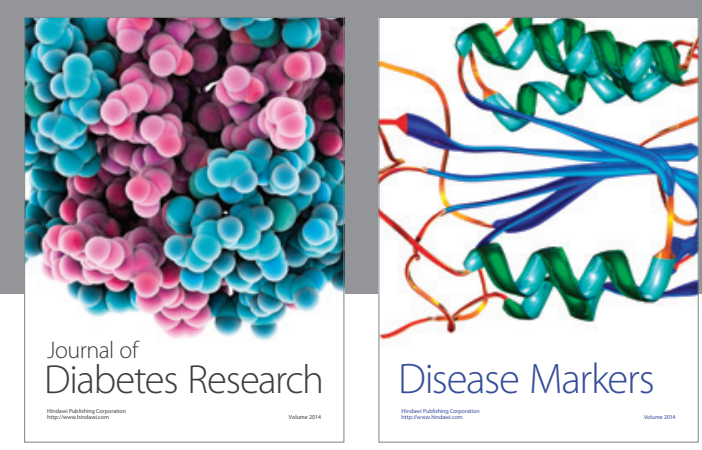

Disease Markers
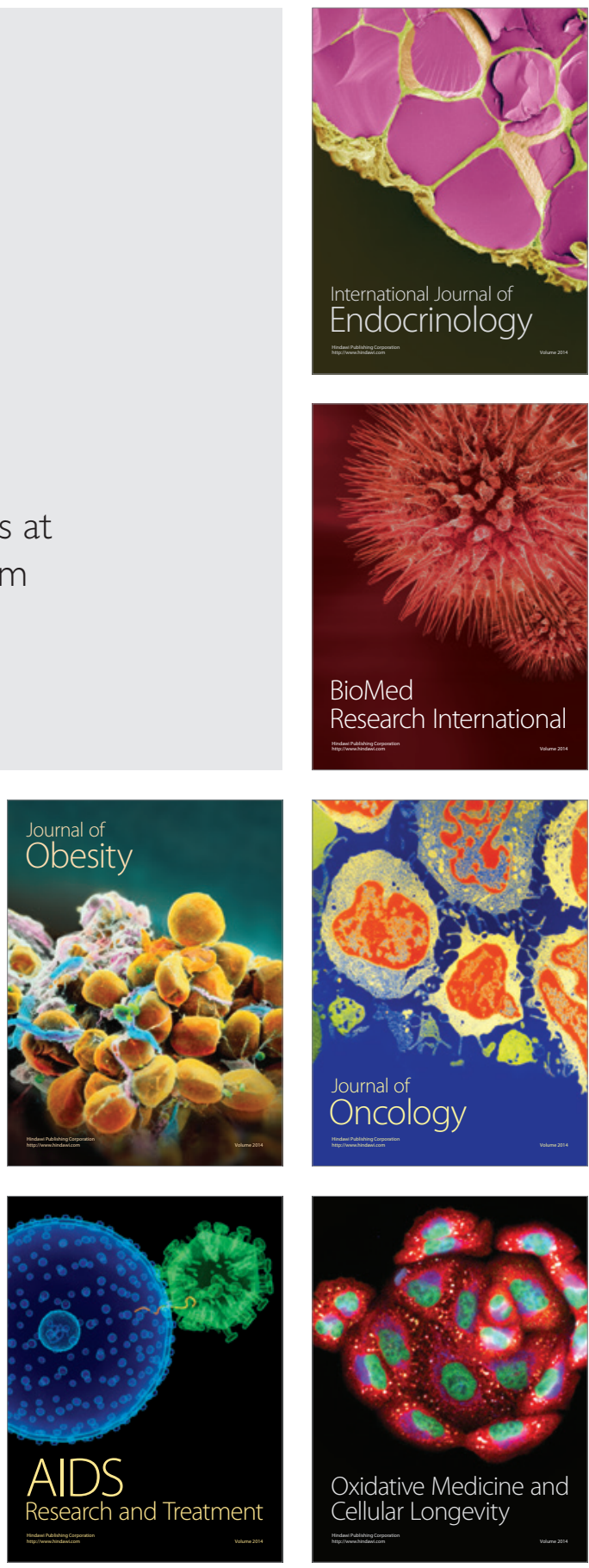